J. Lake Sci. (湖泊科学) , 2015, 27(4):591-598

DOI $10.18307 / 2015.0405$

(C) 2015 by Journal of Lake Sciences

\title{
藻源性湖泛水体显黑颗粒的元素形态分析与鉴定”
}

\author{
申秋实,范成新 ${ }^{* *}$ \\ (中国科学院南京地理与湖泊研究所湖泊与环境国家重点实验室, 南京 210008)
}

\begin{abstract}
摘 要: 湖泛现象是我国超富营养化浅水湖泊面临的最严峻环境问题之一,水体发黑、发臭是湖泛水体最显著和基本的 物理特征. 针对太湖湖泛显黑颗粒缺乏从元素形态直接给出证据的问题, 针对室内模拟藻体和沉积物存在下形成的湖泛 水体, 采用多种图谱分析技术, 对厌氧截留分离的显黑颗粒物质及其化学组成和元素形态进行了分析鉴定. 扫描电镜-X 射线能谱分析与荧光光谱分析表明: 湖泛水体显黑颗粒物质以藻类残体和悬浮沉积物颗粒物为主, 其组成元素以 $\mathrm{C} 、 \mathrm{O} 、 \mathrm{Si}$ 为主; 与未发生湖泛的对照样相比, 湖泛水体显黑颗粒物样品中具有更高的 $\mathrm{Fe} 、 \mathrm{~S}$ 含量. $\mathrm{X}$ 射线光电子能谱 $\mathrm{Fe} 、 \mathrm{~S}$ 微区分 析进一步表明: 湖泛显黑颗粒物样品中 $\mathrm{Fe}$ 主要以 $\mathrm{Fe}(\mathrm{II})$ 的形式存在、S 则以还原态 $\mathrm{S}^{2-}$ 的形式赋存,两者具有共同化合 成 $\mathrm{FeS}$ 的充要条件. 因此, 缺氧厌氧条件下, 湖泛水体的显黑物质组成是 $\mathrm{FeS}$, 它可由水体中 $\mathrm{Fe}^{2+}$ 和 $\Sigma \mathrm{S}^{2-}$ 通过化学共沉淀 形成,并主要通过吸附于悬浮颗粒物之上而使得整个水柱呈现黑色,指示湖泛的暴发过程和维持状态.
\end{abstract}

关键词: 湖泛; 显黑颗粒物; 硫化物

\section{Identification of black suspended particles in the algae-induced black bloom water column}

\section{SHEN Qiushi \& FAN Chengxin}

( State Key Laboratory of Lake Science and Environment, Nanjing Institute of Geography and Limnology, Chinese Academy of Sciences, Nanjing 210008 ,P. R. China)

\begin{abstract}
The black bloom phenomenon is one of the most serious problems in some hyper-eutrophic shallow lakes in China. The black colour and offensive odour were their most significant and basic physical characteristics. However, no firming evidences had been presented to explain what the black causing particles were in the black bloom water. In this research, the algae-induced black bloom was simulated and the chemical compositions of black suspended particles which were intercepted under anaerobic condition after the algae-induced black bloom simulation experiment were analyzed using multi X-ray based approaches. Scanning electron microscope-energy dispersive X-ray spectroscopy and X-ray fluorescence spectrometer analysis showed that algae residues and suspended sediment particles were the main substances and $\mathrm{C}, \mathrm{O}$, and $\mathrm{Si}$ were the main elements of these black particles. Compared to the check samples, black bloom suspended particles performed higher Fe and $\mathrm{S}$ contents. Detailed X-ray photoelectron spectroscopy analysis for $\mathrm{Fe}$ and $\mathrm{S}$ proved that $\mathrm{Fe}$ were mainly in the $\mathrm{Fe}$ ( II ) form while $\mathrm{S}$ were mainly in $\mathrm{S}^{2-}$ form and the majority of them co-existed in the form of FeS. Therefore, in the hypoxic and anoxic black bloom water column, abundant $\mathrm{Fe}^{2+}$ and $\Sigma \mathrm{S}^{2-}$ could co-precipitate into FeS. These black FeS could suspend in the water column or absorb on suspended particles, which caused the black color of the water and marked the formation of the black bloom.
\end{abstract}

Keywords: Black bloom; black causing particle; sulphide

浅水湖泊藻源性湖泛以水体发黑、发臭为最显著的物理特征 ${ }^{[1]}$, 并以此区别于正常湖体, 是我国富营养 化浅水湖泊面临的严重次生水环境灾害之一.自 2007 年由太湖湖泛造成无锡水危机以来,有关湖泛的特 征 ${ }^{[24]}$ 、发生过程 ${ }^{[5-6]}$ 、可能的成因 ${ }^{[7-8]}$ 及其环境影响 ${ }^{[9-11]}$ 和防控措施 ${ }^{[12-13]}$ 等方面均被重点研究. 野外分析及室 内模拟结果均表明: 以二甲基硫醚类物质,如二甲基一硫醚( dimethyl sulfide,DMS)、二甲基二硫醚 (dimethyl

* 国家自然科学基金项目 (51409247)、国家水体污染控制与治理科技重大专项(2012ZX07103-005) 和江苏省太湖水 污染治理专项 (TH2013214) 联合资助. 2014-12-24 收稿;2015-03-10 收修改稿. 申秋实(1982 ), 男, 博士, 助 理研究员;E-mail: junma1202@ sina.com.

** 通信作者;E-mail: cxfan@ niglas. ac.cn. 
disulfide, DMDS)、二甲基三硫梄( dimethyl trisulfide, DMTS) 和甲硫醇 ( methanethiol, MTL) 等为代表的挥发性 有机硫化物 (volatile organic sulfur compounds, VOSCs) 是造成湖泛水体发臭的主要嗅味物 质 ${ }^{[1-2]}$; 蓝藻等生源 性物质的大规模死亡降解是嗅味物质产生的主要途径 ${ }^{[1-2,14-15]}$. 但有关湖泛水体呈现为黑色的原因以假设和 推测居多 ${ }^{[3,7-8]}$, 缺乏足够的直接证据. 然而, 水柱呈现明显的黑色是湖泛水体区别于正常湖体的第一要素, 水柱变黑与否是判断湖泛是否发生的最重要依据 ${ }^{[5]}$. 因此, 研究并分析湖泛水体显黑的原因, 是科学解释湖 泛现象的重要命题.

在自然状态下, 河流、湖泊等原本非黑色地表水体发黑的原因主要有 3 种: 其一, 受污染水体在缺氧戻 氧的环境下生成黑色金属硫化物而发黑, 如美国 Big Pit 湖 ${ }^{[16]}$ 和 Lower Mystic 湖 ${ }^{[17]}$; 其二, 土壤中腐殖质等 深色或黑色有机颗粒物质受长期淋溶作用影响迁移进人水体而使河流、湖泊等变为棕褐直至黑色, 如巴西 亚马逊河流域的 Lago Tupé 湖 ${ }^{[18]}$ 和印度尼西亚苏门答腊的 Siak 河 ${ }^{[19]}$; 其三, 由于某种原生动物或微型后生 动物突然暴发而造成水体视觉上的黑色效应, 如意大利的 Garda 湖 ${ }^{[20]}$. 关于太湖等浅水湖泊藻源性湖泛水 体变黑的原因,不同的研究人员提出不同的假设. 有分析认为: 厌氧还原态环境中形成的黑色表层沉积物在 风浪扰动作用下进人水体, 使得水体呈现黑色 ${ }^{[7,21]}$. 该假设的主要问题在于: 湖泛发生时往往处于平静无风 或微风等物理扰动微小的状态 ${ }^{[8]}$, 不具备所谓底泥上翻的物理条件. 另外一种观点认为: 在湖泛缺氧厌氧的 环境下,水环境中具备形成黑色金属硫化物的客观条件,水体中存在大量以黑色 FeS 为代表的金属硫化物 可能是藻源性湖泛水体显黑的主要原因 ${ }^{[3]}$. 然而, 该观点建立在理论判断的基础上, 缺乏足够的直接证据支 撑. 还有一种观点认为: 湖泛水体中大量增加的有色可溶性有机物 (CDOM) 对光线有一定的吸收, 并通过这 种消光作用使得水体在表观上向暗色靠近 ${ }^{[22]}$, 可能是湖泛水体呈现黑色的原因. 然而, 该观点无法解释野外 和实验室观测到的湖泛黑色物质存在的问题. 因此, 浅水湖泊藻源性湖泛致黑的原因和机理均需要进一步 探讨和明辨.

基于上述背景, 本文以藻源性湖泛显黑颗粒物质为研究对象, 结合一系列基于 $\mathrm{X}$-射线的分析技术对其 进行鉴定与分析, 以期获得浅水湖泊藻源性湖泛显黑颗粒物质的组分特征, 并进一步分析其可能的致黑物 质,为揭示湖泛致黑机理提供一定的理论补充.

\section{1 实验设计与方法}

\section{1 实验设计}

由于藻源性湖泛在野外的发生具有时间和地点上的不确定性, 难以及时发现和跟踪, 因此本研究以室 内模拟实验为基础, 在成功模拟湖泛之后, 分离湖泛水体中黑色颗粒物质, 并进行相关的鉴定与分析. 模拟 实验在湖泊与环境国家重点实验室 Y-型沉积物再悬浮发生模拟装置 ${ }^{[23]}$ 中进行. 该装置拥有 6 个可独立控 制风浪等级的模拟柱, 被成功用于湖泛发生 ${ }^{[6]}$ 及消退 ${ }^{[5]}$ 等过程的模拟与研究. 模拟实验所需对象主要为沉 积物、水样和藻类. 选择藻源性湖泛易发区太湖月亮湾水域为采样点, 利用重力式大口径沉积物采样器采集 深度 $>20 \mathrm{~cm}$ 的原位沉积物柱状样若干, 同时采集上覆水并装于 $25 \mathrm{~L}$ 聚乙烯桶中, 并用浮游生物网现场采集 水华蓝藻并妥善盛装, 所有样品均于当天带回实验室并于 $24 \mathrm{~h}$ 内在 $\mathrm{Y}$-型沉积物再悬浮发生模拟装置中进 行湖泛模拟实验. 实验设置对照和湖泛模拟两组处理, 在每个模拟柱中底部装人 $20 \mathrm{~cm}$ 深带界面水的原位上 层沉积物, 其后无扰动注人原位湖水并控制水深达到与太湖实际水深相似的 $170 \mathrm{~cm}$, 最后在湖泛模拟柱中 添加 $47.5 \mathrm{~g}$ (鲜重) 经滤网过滤过的蓝藻 (对照样不添加) 进行模拟, 所有实验控制条件和参数均参照文献 [5-6]进行.

\section{2 样品处理与分析}

待湖泛稳定发生后, 采集上覆水水样, 在厌氧手套箱中分别利用孔径为 $0.45 \mu \mathrm{m}$ 的玻璃纤维滤膜 (whatman, GF/F) 和中速定量滤膜对水体中颗粒物质进行拦截过滤, 然后将过滤获得的滤膜连同膜上物质在真空 状态下进行冷冻干燥处理. 经冷冻干燥处理后的玻璃纤维滤膜及截留物在液氮环境下低温厌氧保存, 用于 物质形态及致黑敏感物质结合形态分析; 干燥后的定量滤膜及膜上物质则保存在干燥器中, 用于分析颗粒 物质中主要元素含量. 在本研究中, 对照处理中截留的样品记为 “对照”, 湖泛处理中截留的样品记为 “湖泛”。 
对照及湖泛水体截留悬浮颗粒物质形态观察和分析利用场发射扫描电子显微镜 ( SEM, 日立 S-3000N) 与 $X$ 射线能谱仪 (energy dispersive X-ray spectroscopy,EDS) 联用技术即 SEM-EDS 进行,其中 SEM 用于形态 观察, EDS 用于微区元素相对含量分析. 利用 X-射线苂光光谱仪 (X-ray fluorescence spectrometer, XRFS, ARL-9800) 对悬浮颗粒物进行全量分析, 以获得样品中主要主导元素成份及所占份额. 此外, 还利用 X-射线 光电子能谱技术 (X-ray photoelectron spectroscopy, XPS,Thermo Fischer ESCALAB250) 对颗粒物质中致黑敏感 元素化学结合信息进行分析,进一步推导颗粒物中致黑物质的化学赋存形式.

\section{2 结果与分析}

\section{1 显黑颗粒物 SEM-EDS 分析}

湖泊湖泛以水体发黑、发臭为最典型的特征, 水体是否变为黑色是判断湖泛是否发生的依据 ${ }^{[5-8,13]}$. 本研 究湖泛模拟成功后, 截留湖泛水体上覆水颗粒物质如图 1 所示. 在真空抽滤之后, 水体中不同粒径及来源的 悬浮颗粒物质被富集在滤膜之上,与对照样相比,湖泛样品呈现明显的黑色. 在 SEM 放大 2000 倍的情况下, 对照和湖泛样品中均未发现原生动物和微型后生动物存在的证据. SEM-EDS 元素含量分析结果如图 2 和 表 1 所示.

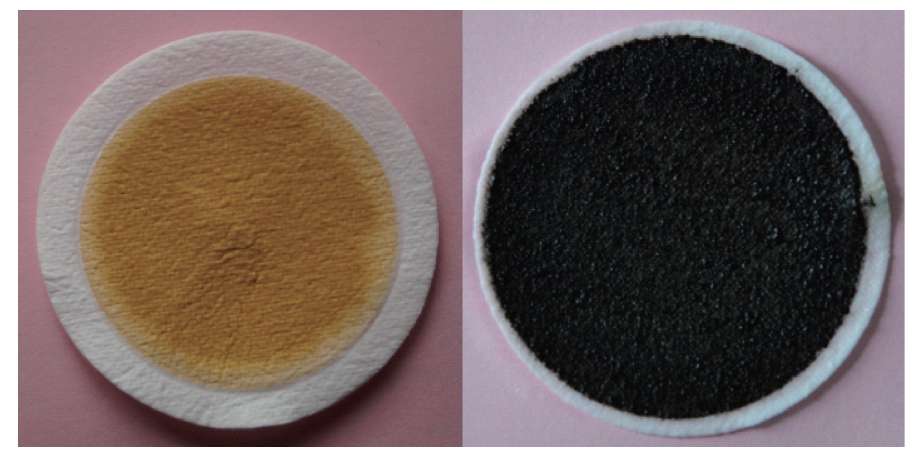

图 1 湖泛水体截留颗粒物质图像(左边为对照样品,右边为湖泛样品)

Fig. 1 Photos of suspended particles of black bloom(left: check; right: black bloom)

表 1 颗粒物质 SEM-EDS 元素含量分析 (At. \%) *

Tab. 1 SEM-EDS element contents analysis of suspended particles

\begin{tabular}{|c|c|c|c|c|c|c|c|c|c|c|c|c|c|c|c|}
\hline 实验组 & & C & 0 & $\mathrm{Si}$ & $\mathrm{Ca}$ & $\mathrm{Fe}$ & $\mathrm{S}$ & $\mathrm{P}$ & $\mathrm{Al}$ & K & $\mathrm{Na}$ & $\mathrm{Mn}$ & $\mathrm{Cu}$ & $\mathrm{Zn}$ & $\mathrm{Mg}$ \\
\hline \multirow[t]{2}{*}{ 对照组 } & (a) & 60.92 & 24.68 & 5.63 & 1.47 & 1.86 & n. d. & 1.05 & 0.52 & 0.32 & 0.48 & 0.21 & 1. 82 & 1.04 & n. d. \\
\hline & (b) & 21.54 & 56.33 & 17. 68 & 0.16 & 0.15 & n. d. & n. d. & 0.71 & n. d. & 2. 13 & n. d. & 0.68 & 0.62 & n. d. \\
\hline \multirow[t]{2}{*}{ 湖泛组 } & (c) & 55.22 & 26.43 & 3. 29 & 0.45 & 2.96 & 4. 86 & n. d. & 1.82 & 0.18 & n. d. & n. d. & 3.79 & 0.65 & 0.34 \\
\hline & (d) & 58.03 & 16. 67 & 8.39 & 0.79 & 3. 18 & 3.89 & n. d. & 0.99 & 0.71 & 1. 16 & n. d. & 3.88 & 2. 31 & n. d. \\
\hline
\end{tabular}

* n. d. 表示未检出,下同.

图 2(a) 为对照样品截留颗粒物致密区 EDS 微区分析, 图 2(b) 为对照样品粒状颗粒类物质 EDS 分析, 结果表明两种不同类型微区元素均以 $\mathrm{C} 、 \mathrm{O} 、 \mathrm{Si}$ 为主. 表 2 显示其主要区别在于图 2(a) 所示致密区 $\mathrm{C}: \mathrm{O}$ 原子 比约为 $2.47: 1$, 图 2(b) 所示粒状颗粒区约为 $0.38: 1$, 且粒状颗粒区 $\mathrm{Si}$ 含量明显高于致密区. 高 $\mathrm{C}: \mathrm{O}$ 原子比 表明相应区域 $\mathrm{C}$ 素主要以有机物形式存在,而二者又是其最主要的组成元素, 因此该部分应以生物质分解 物残体为主. 对应地, 低 $\mathrm{C}: \mathrm{O}$ 原子比表明 $\mathrm{C}$ 素主要以无机形式存在,外加其较高的 $\mathrm{Si}$ 含量, 因此可以认为粒 状颗粒区应以悬浮无机颗粒为主.

图 2(c) 和图 2(d) 分别为湖泛样品截留颗粒物的 EDS 微区分析, 其中图 2(c) 为絮状物质区域、图 2(d) 为致密区. 湖泛样品两类不同类型截留颗粒物均以 $\mathrm{C} 、 \mathrm{O}$ 元素为主, 与对照样相比 $\mathrm{Si}$ 的比重下降较多, 而 $\mathrm{Fe}$ 、 


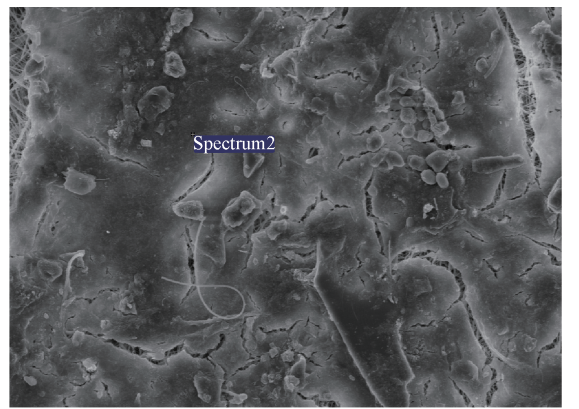

$100 \mu \mathrm{m}$ Electron Image 1

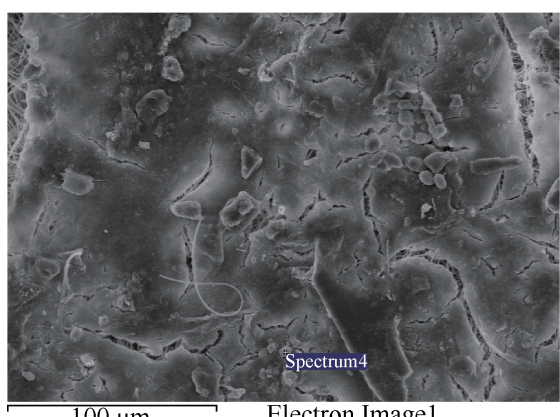

$100 \mu \mathrm{m}$
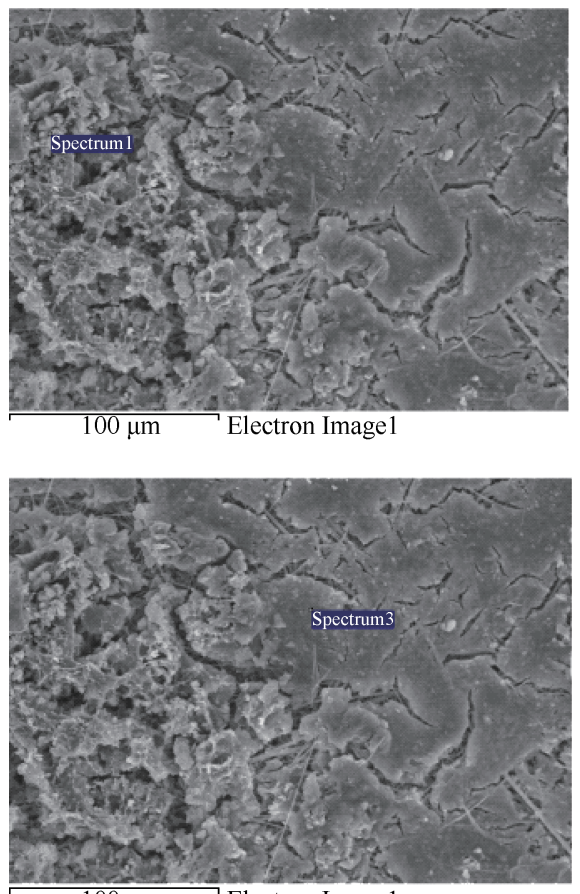

$100 \mu \mathrm{m}$ Electron Image 1
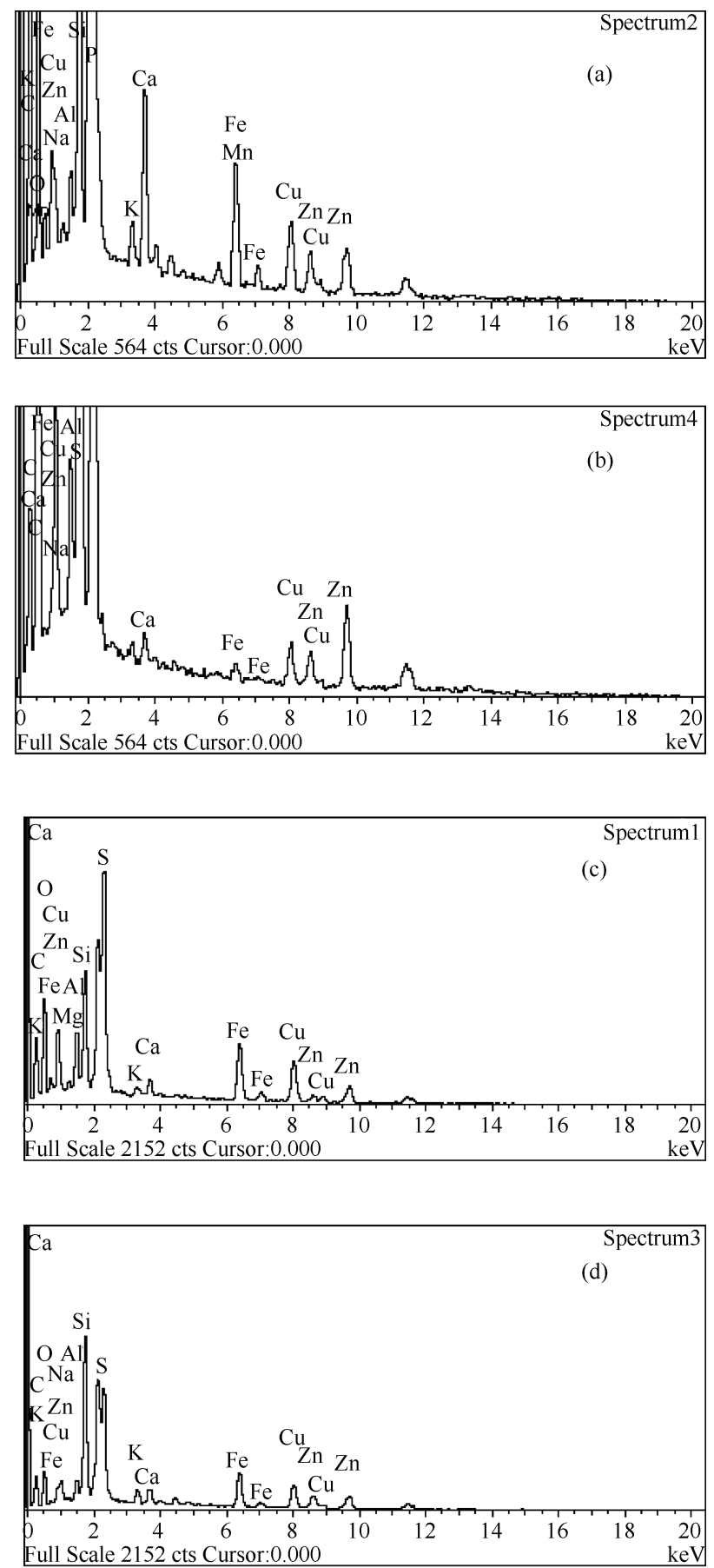

图 2 水体截留颗粒物质 SEM-EDS 分析 ( (a) 和 (b) 为对照样品, (c) 和 (d) 为湖泛样品)

Fig. 2 SEM-EDS analysis of water trapped suspended particles ( (a) and (b) : check; (c) and (d) : black bloom)

$\mathrm{S} 、 \mathrm{Cu}$ 元素所占比例增加明显. 湖泛样品截留颗粒物 $\mathrm{C}: \mathrm{O}$ 比例较高, C 素主要以有机物形式存在, 外加 $\mathrm{C} 、 \mathrm{O}$ 元素是其最主要组成元素 (表 1), 因此, 湖泛样品颗粒物质以蓝藻等生物体分解物残体为主. 值得注意的是, 
与对照样相比湖泛样品中 $\mathrm{Fe} 、 \mathrm{~S}$ 元素所占比例明显上升. 考虑到 $\mathrm{FeS}$ 通常被认为是造成受污染自然水体发黑 的主要物质 ${ }^{[17]}$, 这意味着厌氧还原性环境下悬浮态或吸附于蓝藻等生物质或无机悬浮颗粒物上的黑色 $\mathrm{FeS}$ 沉淀,可能是造成湖泛水体视觉上发黑的重要原因.

\section{2 水体悬浮颗粒物质 XRFS 分析}

对厌氧过滤真空冷冻干燥后的截留颗粒物质进行 XRFS 分析,获得其全量组分信息 (表 2). 烧除有机质 后的湖泛样品及对照样品均以 $\mathrm{Si}$ 作为第一主要组成元素, $\mathrm{Al} 、 \mathrm{P} 、 \mathrm{~K} 、 \mathrm{~N}$ 等常量元素也占有一定的比例. 值得注 意的是, 与黑色 $\mathrm{FeS}$ 相关的 $\mathrm{Fe} 、 \mathrm{~S}$ 两种元素, 其相对含量在对照样品和湖泛样品中具有明显差异.

与对照样相比, 湖泛样品中的 $\mathrm{Fe}$ 和 $\mathrm{S}$ 的含量明显更高. 这说明除颗粒物质自身本底值外, 湖泛样品颗 粒物质上还汇集了外来 $\mathrm{Fe} 、 \mathrm{~S}$ 类物质. 湖泛水体中具有不同价态含量较高的 $\mathrm{Fe} 、 \mathrm{~S}$ 类物质 ${ }^{[1]}$, 这为水体黑色 颗粒物中较高含量的 $\mathrm{Fe} 、 \mathrm{~S}$ 提供来源. 其富集途径可能主要有二: 其一, 湖泛水体中相对丰富的离子态或胶 体态 $\mathrm{Fe} 、 \mathrm{~S}$ 类物质直接吸附于悬浮颗粒物表面,使得湖泛黑色颗粒物中 $\mathrm{Fe}$ 和 $\mathrm{S}$ 含量升高; 其二, 湖泛厌氧水 体中的 $\mathrm{Fe}^{2+}$ 和 $\Sigma \mathrm{S}^{2-}$ 通过化学共沉淀形成黑色 $\mathrm{FeS}$, 该物质或自成颗粒悬浮于水体或吸附沉降在原有悬浮颗 粒物质表面并使得颗粒物成为黑色 $\mathrm{FeS}$ 的载体,从而造成湖泛样品颗粒物中 $\mathrm{Fe}$ 和 $\mathrm{S}$ 含量升高.

表 2 水体悬浮颗粒物质 XRFS 全量分析 ( $\mathrm{w} \%$ )

Tab. 2 XRFS analysis of suspended particles in the water

\begin{tabular}{ccccccccc}
\hline 实验组 & $\mathrm{SiO}_{2}$ & $\mathrm{SO}_{3}$ & $\mathrm{Al}_{2} \mathrm{O}_{3}$ & $\mathrm{P}_{2} \mathrm{O}_{5}$ & $\mathrm{~K}_{2} \mathrm{O}$ & $\mathrm{Na}_{2} \mathrm{O}$ & $\mathrm{Fe}_{2} \mathrm{O}_{3}$ & 烧失量及其它 \\
\hline 对照组 & 56.15 & 3.85 & 5.43 & 6.75 & 4.29 & 2.83 & 1.45 & 19.24 \\
湖泛组 & 47.79 & 14.47 & 5.57 & 5.04 & 4.11 & 3.49 & 2.49 & 17.04 \\
\hline
\end{tabular}

\section{3 湖泛显黑悬浮颗粒物质 XPS 分析}

对截留获得的湖泛样品及对照样品颗粒物质分别进行 XPS 分析, 结果如图 3 及表 3 所示. 与对照样相 比, 湖泛样具有明显的 Fe 元素特征峰 (图 3). 主要元素相对含量分析结果进一步表明, 颗粒物中元素组成以 $\mathrm{C} 、 \mathrm{O} 、 \mathrm{~N} 、 \mathrm{Si}$ 为主, $\mathrm{Fe} 、 \mathrm{~S}$ 也占有一定的比例 (表 3). 两类样品中 $\mathrm{C}: \mathrm{O}$ 原子比均较高, 这与 SEM-EDS 分析得出的 结果基本吻合. 与对照样相比, 湖泛水体颗粒物中 $\mathrm{Fe}$ 和 $\mathrm{S}$ 含量明显较高, 二者分别比对照样高 10.6 倍和 3.5 倍.

表 3 水体悬浮颗粒物质元素组成 XPS 分析

Tab. 3 XPS element contents analysis of suspended particles in the water

\begin{tabular}{rcccccccc}
\hline \multirow{2}{*}{ 实验组 } & \multicolumn{7}{c}{ 元素相对含量 $/($ At. \% ) } \\
\cline { 2 - 9 } & $\mathrm{C}$ & $\mathrm{O}$ & $\mathrm{N}$ & $\mathrm{Fe}$ & $\mathrm{S}$ & $\mathrm{Si}$ & $\mathrm{Mn}$ \\
\hline 对照组 & 60.25 & 29.86 & 7.57 & 0.1 & 0.26 & 1.54 & 0.11 \\
湖泛组 & 58.78 & 30.28 & 7.21 & 1.06 & 0.92 & 1.06 & n. d. \\
\hline
\end{tabular}

在自然水体黑臭现象中,黑色 $\mathrm{FeS}$ 或金属硫化物被认为是很多受污染黑臭水体发黑的直接原因 ${ }^{[17]}$. 通 过一系列分析表明,在湖泛黑色颗粒截留物中 $\mathrm{Fe} 、 \mathrm{~S}$ 含量明显高于对照样. 然而,这两种元素在自然界中赋 存形态多样且化合形式复杂多变,尤其 $S$ 元素具有丰富的化合价. 因此,湖泛样品中 $\mathrm{Fe}$ 和 $\mathrm{S}$ 元素以及其它可 形成黑色金属硫化物的元素以何种形式赋存,对湖泛黑色颗粒物显黑原因具有重要意义. 为此,对颗粒物样 品针对 $\mathrm{Hg} 、 \mathrm{~Pb} 、 \mathrm{Mn} 、 \mathrm{Fe} 、 \mathrm{~S}$ 等元素利用 XPS 进行特征峰微区分析. 分析显示, $\mathrm{Hg} 、 \mathrm{~Pb} 、 \mathrm{Mn}$ 这 3 种元素含量低于 可检出光子数,没有出现明显的特征峰, $\mathrm{S}$ 和 $\mathrm{Fe}$ 含量较为丰富,特征峰明显.

颗粒物中 S 元素 XPS 微区分析结果如图 4a 所示. 与对照样品相比, 湖泛水体颗粒物样品中 S 含量更加 丰富, 其 $S 2 p$ 谱线具有更强的特征峰. 分峰拟合之后可以清楚地看到, 湖泛样品中 $S$ 的赋存形式以还原态 $S$ 为主, 里面包含有 $\mathrm{S}^{2-} 、 \mathrm{~S}_{2}^{2-} 、 \mathrm{HS}^{-} 、 \mathrm{~S}_{8} 、 \mathrm{~S}_{0}$ 等一些列终端还原态或中间还原态产物. 最强特征峰出现在结合能约 为 $161.3 \mathrm{eV}$ 处,该处 $S$ 元素主要以 $S^{2-}$ 化合态形式存在 ${ }^{[24]}$,这表明以硫化物形式存在的 $S^{2-}$ 在湖泛截留颗粒 物 $\mathrm{S}$ 中占有最多的份额. 由于除了 $\mathrm{Fe}$ 之外, 并无足够的其它金属元素可以与 $\mathrm{S}$ 形成硫化物, 因此, 在湖泛样 


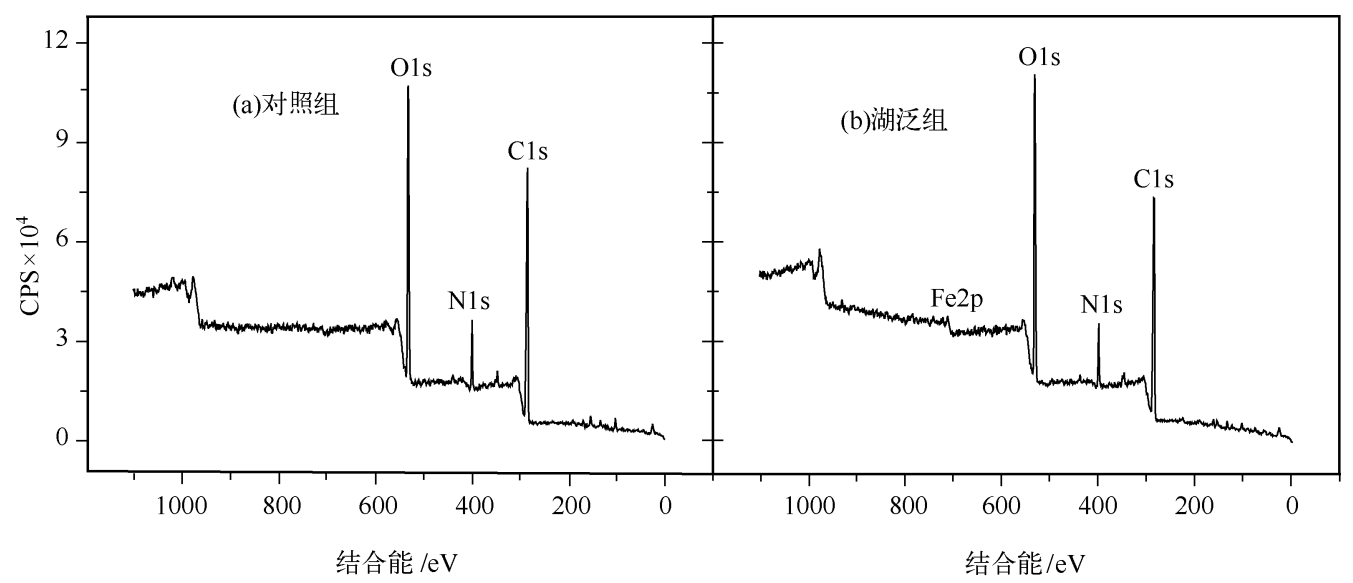

图 3 湖泛水体悬浮颗粒物 XPS 图谱

Fig. 3 XPS analysis of suspended particles in black bloom water

品中这部分 $\mathrm{S}$ 主要是以 $\mathrm{FeS}$ 的形式存在. 形成鲜明对比的是, 对照样中 $\mathrm{S}$ 的存在形式以 $\mathrm{SO}_{4}^{2-}$ 为主, 以还原态 形式存在的 S 只占据很小的比例,基本可以忽略不计.

颗粒物中 Fe 元素 XPS 微区分析结果如图 4b 所示. 湖泛样品与对照样颗粒物质 Fe $2 \mathrm{p}$ 能谱线具有相似 的变化特征, 均呈典型的马鞍形特征峰. 由于 $\mathrm{Fe}$ 的化合物繁多, Fe $2 \mathrm{p} 3 / 2$ 轨道的电子具有非常多且间隔很 小的峰位, 因此对于来自自然环境中成分复杂的样品我们很难将其一一区分出. 对 XPS 谱图分析后发现, 湖 泛样品在大约 713.6 .712.1、710.3 eV 处有小的特征峰出现, 根据数据库 ${ }^{[25]}$ 资料对比发现, 这些特征峰应该 为 $\mathrm{FeS}$ 类物质, 这与 $\mathrm{S}$ 元素微区分析结果相吻合. 对照样品在大约 $713.3 \mathrm{eV}$ 附近出现较弱的 $\mathrm{Fe}^{3+}$ 特征峰, 对 比数据库资料并结合该样品 $\mathrm{S}$ 元素微区分析结果, 可以判定该样品中 $\mathrm{Fe}$ 与 $\mathrm{S}$ 主要以 $\mathrm{Fe}_{2}\left(\mathrm{SO}_{4}\right)_{3}$ 的方式 赋存.
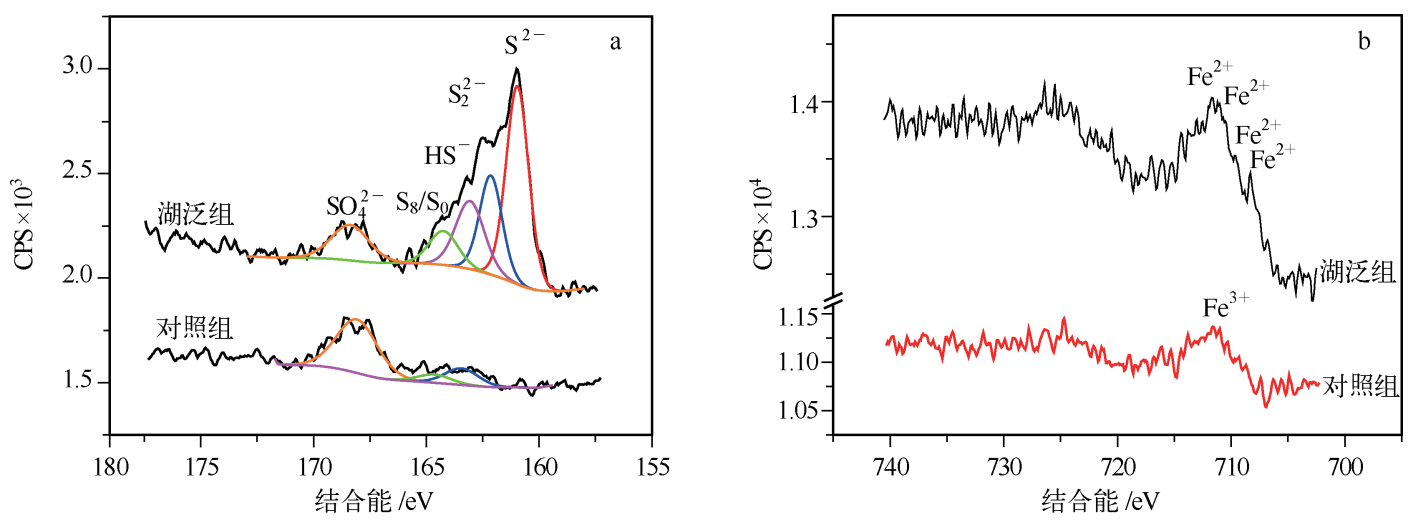

图 4 截留悬浮颗粒物 $\mathrm{S}$ 元素 (a) 和 $\mathrm{Fe}$ 元素( b)XPS 微区分析

Fig. 4 Detailed S (a) and Fe (b) XPS analysis of suspended particles

\section{3 讨论}

湖泛黑臭水体的形成与湖泊富营养化紧密相关, 与受污染黑臭河流等黑臭水体既具有一定的共性又存 在明显差别. 城市黑臭河道大多受居民生活用水和市政污水的影响, 水体长期处于高生化需氧量、高化学需 氧量和高有机负荷的状态, 其水体发黑被认为主要是金属硫化物增加、有机质腐殖化以及污染底泥再悬浮 
共同造成的结果 ${ }^{[19,26-28]}$. 湖泛的发生与蓝藻的大规模暴发和积聚有关 ${ }^{[1-10]}$, 在发生湖泛前水体处于富营养化 湖泊的常规状态, 其最终暴发是大型水体缺氧现象发展第 4 个阶段的必然结果. 在该阶段,大量积聚的水华 藻类死亡降解并引起水体和表层沉积物缺氧而形成还原性环境 ${ }^{[29]}$, 水环境中 $\mathrm{Fe}^{3+}$ 失去稳定存在的依据而 不断被还原为 $\mathrm{Fe}^{2+}$, 同时硫酸盐还原菌 ( sulfate reducing bacteria, $\mathrm{SRB}$ ) 开始增殖并代谢还原高价态的 $\mathrm{SO}_{4}^{2-}$ 而生成终端还原产物 $\mathrm{H}_{2} \mathrm{~S}^{[1,26]}$, 给 $\mathrm{FeS}$ 致黑物质的形成提供了初始的物质条件. 野外原位观测 ${ }^{[1]}$ 和室内等比 例模拟 ${ }^{[13]}$ 均良好地印证了湖泛致黑物质物源基础的假设.

与野外实际情况相比, 室内湖泛模拟实验尽量控制为理想条件, 获得的湖泛水色更黑、臭味更强烈暨发 生程度往往更深一些,湖泛体系组成也较野外实际情况简单,但却有利于湖泛致黑致臭机理研究. 本研究湖 泛水体黑色悬浮颗粒物 SEM-EDS 和 XRFS 分析结果表明: 水柱黑色颗粒态物质表面 $\mathrm{Fe} 、 \mathrm{~S}$ 含量较高, 其原子 比通常接近 $1: 1$. 在湖泛水体中, 较高的 $\mathrm{Fe}^{2+}$ 和 $\Sigma \mathrm{S}^{2-}$ 含量是其水环境的重要化学特征之一 ${ }^{[1]}$. 在湖泛这样的 厌氧环境中, 这类 $\mathrm{Fe} 、 \mathrm{~S}$ 物质极有可能通过化学共沉淀作用形成黑色 $\mathrm{FeS}$, 其会以细微颗粒存在于水柱中、或 者吸附于较大悬浮颗粒物之上, 从而使得水体呈现视觉上的黑色. XPS 分析进一步表明,在湖泛水体黑色悬 浮颗粒物中, $\mathrm{S}$ 主要以 $\mathrm{S}^{2-}$ 硫化物的形式存在, $\mathrm{Fe}$ 主要以 $\mathrm{Fe}(\mathrm{II})$ 还原态硫化物 $\mathrm{FeS}$ 的形式存在. 由于颗粒物 质样品中并未出现除 $\mathrm{Fe}$ 以外的其它足够含量可与 $\mathrm{S}^{2-}$ 形成黑色硫化物的金属元素, 外加 XPS 分析结果显示 $\mathrm{Fe}: \mathrm{S}$ 原子比基本为 $1: 1$, 由此可以确定湖泛黑色悬浮颗粒物中 $\mathrm{Fe}$ 和 $\mathrm{S}$ 主要以 $\mathrm{FeS}$ 的化合形式存在. 因此,综 合多种分析认为:厌氧条件下形成的黑色 $\mathrm{FeS}$ 是引起湖泛水体发黑的重要原因,该类物质或悬浮于水柱或 吸附在水体悬浮颗粒物质之上而广泛分布于湖泛水体,从而造成湖泛水体强烈的黑色视觉效应.

水柱中黑色颗粒物在湖体中稳定存在与否,指示了湖泛的发生、维持与消退. 对于具有水深较深且存在 水温季节性分层的大型水体,黑水或缺氧/厌氧层能稳定存在于从春季到秋季的较长时间尺度 ${ }^{[1,30]}$. 但对太 湖湖泛野外调查跟踪结果显示, 湖泛黑色水体持续时间从 $1 \mathrm{~d}$ 到 $25 \mathrm{~d}$ 不等 ${ }^{[31]}$, 不同时间和地点发生的湖泛 持续时间差异明显. 公认的解释是: 水体保持缺氧厌氧状态是黑色 $\mathrm{FeS}$ 类致黑物质稳定存在的前提与基 础 $^{[16-17]}$. 一旦这种还原态环境状态被破坏, 水柱中黑色 $\mathrm{FeS}$ 将被氧化消耗, 从而使得湖泛黑色水体消失且水 色回归正常. 在自然湖体中, 风浪扰动造成的水体复氧效应非常重要, 在风浪持续作用下, 水体复氧能力加 强并造成湖泛水体从还原态环境向氧化态环境转化, 使得湖泛水体 $\mathrm{FeS}$ 类致黑物质最终受氧化而消失, 进 而造成湖泛水体的恢复 ${ }^{[5]}$, 是湖泛黑色水体消失的主要原因. 相反, 在水动力条件稳定的条件下, 水体缺氧 厌氧状态不易被打破,黑色 $\mathrm{FeS}$ 类物质在该条件下继续稳定存在, 湖泛黑色水体得以保持 ${ }^{[5]}$.

\section{4 结论}

在室内模拟藻源性湖泛的基础上, 分离截留湖泛水体显黑颗粒物质,并借助 SEM-EDS、XRFS、XPS 等一 系列基于 X 射线的分析手段,对湖泛样品显黑颗粒物质进行分析. SEM-EDS 和 XRFS 分析结果显示,湖泛水 体显黑颗粒物质以生物质残体和悬浮沉积物颗粒为主. 与非湖泛对照样相比, 其主要化学组分除 $\mathrm{C} 、 \mathrm{~N} 、 \mathrm{O} 、 \mathrm{Si}$ 外 还含有含量较高的 Fe、S. XPS 分析进一步表明,湖泛颗粒中 $\mathrm{Fe} 、 \mathrm{~S}$ 主要以还原态形式存在,二者大量地以 $\mathrm{FeS}$ 的化合形式共存,这是湖泛水体颗粒物质呈现黑色的最主要原因. 可以认为, 在湖泊水体缺氧厌氧环境下, 当 氧化还原环境适宜时, 水体中丰富的 $\mathrm{Fe}^{2+}$ 和 $\Sigma \mathrm{S}^{2-}$ 通过化学共沉淀作用化合成黑色 $\mathrm{FeS}$ 而广泛分布于水体和悬 浮颗粒物中, 从而造成湖泛的暴发和持续. 然而, 有关致黑物质反应物来源和迁移转化过程目前尚不清楚, 还 需进一步跟进研究,为科学解释湖泛发生机理及合理预控湖泛提供更深人的理论基础.

\section{5 参考文献}

[ 1 ] Shen QS, Zhou QL, Shang JG et al. Beyond hypoxia: Occurrence and characteristics of black blooms due to the decomposition of the submerged plant Potamogeton crispus in a shallow lake. Journal of Environmental Sciences, 2014, 26 (2): 281-288.

[ 2 ] Yang M, Yu JW, Li ZL et al. Taihu Lake not to blame for Wuxi's woes. Science, 2008, 319(5860) : 158.

[ 3 ] 刘国锋,钟继承,何 俊等. 太湖竺山湾藻华黑水团沉积物中 Fe-S-P 的含量及其形态变化. 环境科学, 2009, 30 (9) : 2520-2526.

[ 4 ] 中国科学院南京地理与湖泊研究所. 太湖梅梁湾 2007 年蓝藻水华形成及取水口污水团成因分析与应急措施建 
议. 湖泊科学, 2007, 19(4): 357-358.

[ 5] 申秋实, 邵世光, 王兆德等. 风浪条件下太湖藻源性 “湖泛” 的消退及其水体恢复进程. 科学通报, 2012, 57(12): 1060-1066.

[6] 申秋实, 邵世光, 王兆德等. 太湖月亮湾湖泛发生过程模拟及水土物化性质的响应. 水科学进展, 2011, 22(5): 710-719.

[ 7 ] 陆桂华,马 倩. 太湖水域“湖泛”及其成因研究. 水科学进展, 2009, 20(3): 438-442.

[8] 陈荷生.太湖宜兴近岸水域“湖泛”现象初析. 水利水电科技进展, 2011, 31(4) : 33-37.

[9] 刘国锋, 申秋实, 张 雷等. 藻源性黑水团环境效应: 对水一沉积物界面氮磷变化的驱动作用. 环境科学, 2010, 31 (12) : 2917-2924.

[10］刘国锋,何 俊,范成新等. 藻源性黑水团环境效应: 对水一沉积物界面处 Fe、Mn、S 循环影响. 环境科学, 2010,31 (11) : 2652-2660.

[11] 申秋实, 周麒麟, 邵世光等. 太湖草源性“湖泛” 水域沉积物营养盐释放估算. 湖泊科学, 2014, 26(2): 177-184.

[12] He W, Shang JG, Lu X et al. Effects of sludge dredging on the prevention and control of algae-caused black bloom in Taihu Lake, China. Journal of Environmental Sciences, 2013, 25(3) : 430-440.

[13] Shen QS, Liu C, Zhou QL et al. Effects of physical and chemical characteristics of surface sediments in the formation of shallow lake algae-induced black bloom. Journal of Environmental Sciences, 2013, 25(12) : 2353-2360.

[14] Lu X, Fan CX, Shang JG et al. Headspace soli-phase microextraction for the determination of volatile sulfur compounds in odorous hyper-eutrophic freshwater lakes using gas chromatography with flame photometric detection. Microchemical Journal, 2012, 104: 26-32.

[15] 卢 信, 冯紫燕, 商景阁等. 不同有机质诱发的水体黑臭及主要致臭物 (VOSCs) 产生机制研究. 环境科学, 2012, $33(9)$ : 3152-3159.

[16] Stahl JB. Black water and 2 peculiar types of stratification in an organically loaded strip-mine lake. Water Research, 1979, $13(5)$ : $467-471$.

[17] Duval B, Ludlam SD. The black water chemocline of meromictic Lower Mystic Lake, Massachusetts, USA. International Review of Hydrobiologia, 2001, 86(2) : 165-181.

[18 ] Rai H, Hill G. Physical and chemical studies of lago tupe- a central amazonian black water ria lake. Internationale Revue Der Gesamten Hydrobiologie, 1981, 66(1) : 37-82.

[19] Rixen T, Baum A, Pohlmann T et al. The Siak, a tropical black water river in central Sumatra on the verge of anoxia. Biogeochemistry, 2008, $90(2)$ : 129-140.

[20] Pucciarelli S, Buonanno F, Pellegrini G et al. Biomonitoring of Lake Garda: Identification of ciliate species and symbiotic algae responsible for the "black-spot" bloom during the summer of 2004. Environmental Research, 2008, 107 (2): 194-200.

[21］王成林,张 咏,张宁红等.太湖藻源性“湖泛”形成机制的气象因素分析. 环境科学, 2011, 32(2): 401-408.

[22] Duan HT, Ma RH, Loiselle SA et al. Optical characterization of black water blooms in eutrophic waters. Science of the Total Environment, 2014, 482 :174-183.

[23］范成新. 一种室内模拟水下沉积物再悬浮状态的方法及装置: 中国, 200410014329. X X P ]. 2005-01-12.

[24] Shchukarev A, Galman V, Rydberg J et al. Speciation of iron and sulphur in seasonal layers of varved lake sediment: an XPS study. Surface and Interface Analysis, 2008, 40(3/4): 354-357.

[25] National Institute of Standards and Technology. NIST X-ray Photoelectron Spectroscopy Database 20, Version 4. 1. In http ://srdata. nist. gov/xps/, 2012.

[26] 钱嫦萍,王启东,陈振楼等.生物修复技术在黑臭河道治理中的应用. 水处理技术, 2009, 35(4): 13-17.

[27] 雷恒毅, 余光伟, 刘立光等. 珠江流域中污染感潮河道黑臭治理新技术. 中山大学学报: 自然科学版, 2007, 46(3): 134-136.

[28] 钱嫦萍,陈振楼,王启东. 城市河流黑臭的原因分析及生态危害. 城市环境, 2002, 16(3): 21-23.

[29] Diaz RJ, Rosenberg R. Spreading dead zones and consequences for marine ecosystems. Science, 2008, 321 (5891): 926-929.

[30 ] Turner RE, Rabalais NN, Swenson EM et al. Summer hypoxia in the northern Gulf of Mexico and its prediction from 1978 to 1995. Marine Environmental Research, 2005, 59(1): 65-77.

[31］陆桂华, 马 倩. 2009 年太湖水域“湖泛” 监测与分析. 湖泊科学, 2010, 22(4): 481-487. 